\title{
Polariton Anomalous Hall Effect in Transition-Metal Dichalcogenides
}

DOI:

10.1103/PhysRevLett.121.137402

\section{Document Version}

Final published version

Link to publication record in Manchester Research Explorer

\section{Citation for published version (APA):}

Gutiérrez-rubio, Á., Chirolli, L., Martín-moreno, L., García-vidal, F. J., \& Guinea, F. (2018). Polariton Anomalous Hall Effect in Transition-Metal Dichalcogenides. Physical Review Letters, 121(13), [137402].

https://doi.org/10.1103/PhysRevLett.121.137402

\section{Published in:}

Physical Review Letters

\section{Citing this paper}

Please note that where the full-text provided on Manchester Research Explorer is the Author Accepted Manuscript or Proof version this may differ from the final Published version. If citing, it is advised that you check and use the publisher's definitive version.

\section{General rights}

Copyright and moral rights for the publications made accessible in the Research Explorer are retained by the authors and/or other copyright owners and it is a condition of accessing publications that users recognise and abide by the legal requirements associated with these rights.

\section{Takedown policy}

If you believe that this document breaches copyright please refer to the University of Manchester's Takedown Procedures [http://man.ac.uk/04Y6Bo] or contact uml.scholarlycommunications@manchester.ac.uk providing relevant details, so we can investigate your claim.

\section{OPEN ACCESS}




\title{
Polariton Anomalous Hall Effect in Transition-Metal Dichalcogenides
}

\author{
Á. Gutiérrez-Rubio, ${ }^{1, *}$ L. Chirolli, ${ }^{1, \dagger}$ L. Martín-Moreno, ${ }^{2}$ F. J. García-Vidal, ${ }^{3,4}$ and F. Guinea ${ }^{1,4,5}$ \\ ${ }^{1}$ IMDEA Nanoscience Institute, C/Faraday 9, E-28049 Madrid, Spain \\ ${ }^{2}$ Departamento de Física de la Materia Condensada, Instituto de Ciencia de Materiales, Universidad de Zaragoza, \\ E-50009 Zaragoza, Spain \\ ${ }^{3}$ Departamento de Física Teórica de la Materia Condensada and Condensed Matter Physics Center (IFIMAC), \\ Universidad Autónoma de Madrid, E-8049 Madrid, Spain \\ ${ }^{4}$ Donostia International Physics Center (DIPC), E-20018 Donostia/San Sebastián, Spain \\ ${ }^{5}$ School of Physics and Astronomy, University of Manchester, Manchester M13 9PY, United Kingdom
}

(Received 10 February 2018; published 26 September 2018)

\begin{abstract}
We analyze the properties of strongly coupled excitons and photons in systems made of semiconducting two-dimensional transition-metal dichalcogenides embedded in optical cavities. Through a detailed microscopic analysis of the coupling, we unveil novel, highly tunable features of the spectrum that result in polariton splitting and a breaking of light-matter selection rules. The dynamics of the composite polaritons is influenced by the Berry phase arising both from their constituents and from the confinementenhanced coupling. We find that light-matter coupling emerges as a mechanism that enhances the Berry phase of polaritons well beyond that of its elementary constituents, paving the way to achieve a polariton anomalous Hall effect.
\end{abstract}

DOI: 10.1103/PhysRevLett.121.137402

Introduction.-The motion of composite excitations of quantum systems requires the introduction of gauge potentials related to their internal structure [1], encoded in the Berry curvature (BC) [2] in momentum space. A generalization to periodic systems has led to a deep understanding of quantized topological properties and edge modes $[3,4]$. Nontrivial Berry phases have been extensively discussed for electrons, photons [5], and excitons [6], among others [7,8], and the concept of Hall effect has been extended to both photons $[9,10]$ and excitons [11,12]. Additionally, systems with strong light-matter interactions are being intensively studied, as their properties can be tuned through coupling to light [13]. Two-dimensional materials, such as transition-metal dichalcogenides (TMDs) [14,15] embedded in optical cavities, stand out as an excellent platform where strong light-matter interactions can be studied [16-18]. Moreover, their band structures bring about nontrivial topological features. Those of excitons coupled to photons in quasi-two-dimensional geometries have been first pointed out in Refs. [19-21], where a winding phase in the coupling was recognized as the main ingredient to construct topological polaritonic crystals. Reference [22] is also remarkable in this respect, carrying out a thorough analysis in terms of the geometric tensor.

In this work, we study strongly coupled excitons and photons in a monolayer of $\mathrm{MoS}_{2}$ embedded in a cavity. We focus on the role of the composite Berry curvature, arising from both the bare constituents and their coupling, on the motion of the composite excitations, and the possibility to induce a polariton anomalous Hall effect. Through a detailed microscopic analysis, we show that the cavity breaks the polarization-valley locking [23,24], which manifests in a cross-coupling between right (left) circularly polarized photons and valley $K\left(K^{\prime}\right)$ excitons, characterized by a winding phase. This gives rise to a fine splitting of the upper and lower polaritons. We then analyze the impact of the winding coupling in the $\mathrm{BC}$ of the composite polaritons and show that it yields values for the hybrid modes far greater than those of the exciton and photon taken separately, showing that light-matter interaction dominates the $\mathrm{BC}$ in the strong-coupling regime. By carrying out a semiclassical analysis of polaritonic wave packets, we estimate measurable topological Hall drifts, which may shed light on recent experiments and pave the way to achieve a polariton anomalous Hall effect in TMDs.

The model.-We consider excitons in a $\mathrm{MoS}_{2}$ monolayer embedded in a planar optical cavity (see the inset in Fig. 1). The maximum (minimum) of the valence (conduction) bands of a $\mathrm{MoS}_{2}$ monolayer lies at the two nonequivalent corners of the hexagonal Brillouin zone, $K$ and $K^{\prime}$. The valence band shows a significant spin-orbit splitting, $\Delta_{\mathrm{SO}} \sim 100 \mathrm{meV}$, resulting in a spin-valley locking. Excitons in opposite valleys, related by time-reversal symmetry, have opposite spin. In the absence of a magnetic field, they are degenerate. Photons in perfect conducting cavities are also doubly degenerate (except for electric fields perpendicular to the monolayer, not coupled to excitons). This motivates a simplified model which includes two excitons and the two photons closest in energy to the excitons. We assume perfect translational symmetry, so excitons and photons have a 
well-defined parallel momentum q. Remarkably, the valleypolarization selection rule $[23,24]$ holds only at $\mathbf{q}=0$.

We describe excitons with momentum $\mathbf{q}$ in valley $\tau$ through a variational Wannier wave function [25]

$$
\left|\psi_{\mathrm{ex}}^{\tau}(\mathbf{q})\right\rangle=\int d^{2} q^{\prime} \phi\left(q^{\prime}\right)\left[c_{\mathbf{q}^{\prime}+(\mathbf{q} / 2)}^{\tau}\right]^{\dagger} v_{\mathbf{q}^{\prime}-(\mathbf{q} / 2)}^{\tau}|0\rangle .
$$

Here, $c_{\mathbf{q}}^{\tau}\left(v_{\mathbf{q}}^{\tau}\right)$ destroys a conduction (valence) electron in valley $\tau$ with wave vector $\mathbf{q}=\left(q_{x}, q_{y}\right),|0\rangle$ denotes the filled Fermi sea, and $\phi(q)=\sqrt{(2 / \pi)} a_{\mathrm{ex}}\left[1+\left(k a_{\mathrm{ex}}\right)^{2}\right]^{-3 / 2}$ is the $s$-wave exciton wave function describing the relative motion of the bound electron and hole. The variational parameter $a_{\mathrm{ex}}$ is the average radius of the exciton, which can be approximated by the typical value of $1 \mathrm{~nm}$ for $\mathrm{MoS}_{2}$ $[26,27]$. Our model therefore deals with Wannier excitons of small radii. This point is relevant when studying 2D materials like $\mathrm{MoS}_{2}$, where dielectric screening changes significantly with respect to the 3D case. For an in-depth analysis of the excitonic excited states, see Refs. [28,29]. We omit these in our work due to a parity mismatch that prevents their coupling with single photons.

In the continuum limit and defining $\left|\psi_{\mathrm{ex}}^{\tau}(\mathbf{q})\right\rangle=\left[b_{\mathbf{q}}^{\tau}\right]^{\dagger}|0\rangle$, the bare excitonic Hamiltonian reads

$$
H_{\mathrm{ex}}=\sum_{\tau} \int d^{2} q\left(\frac{\hbar^{2} q^{2}}{2 M_{\mathrm{ex}}}+2 \Delta+E_{b}\right)\left[b_{\mathbf{q}}^{\tau}\right]^{\dagger} b_{\mathbf{q}}^{\tau}
$$

with an exciton mass $M_{\mathrm{ex}}=m_{e}+m_{h} \simeq 0.74 m_{0}\left(m_{e}\right.$ and $m_{h}$ being the masses of the bound electron and hole, respectively, and $m_{0}$ the electron rest mass), half gap $\Delta \simeq 1.5 \mathrm{eV}$, and binding energy $E_{b} \simeq-1.1 \mathrm{eV}[30,31]$.

Confined electromagnetic modes in a cavity with perfectly conducting mirrors and height $L_{z}$ have a momentum $\mathbf{k}=\left(\mathbf{q}, k_{z}\right)$, with $\mathbf{q}=\left(q_{x}, q_{y}\right)$ and $k_{z}=\pi m / L_{z}$, with $m$ an integer. We neglect the effect of fields associated to surface plasmons at the metallic boundaries of the cavity, as $L_{z} \simeq \lambda / 2$, and also disregard modes with electric fields parallel to $\mathbf{e}_{z}$, as they do not couple to excitons in the $\mathrm{MoS}_{2}$ layer. In the basis of circularly polarized light, for which we define the bosonic operators $a_{\mathbf{q}, k_{z}}^{\nu}$ with polarizations $\nu= \pm$, the photonic Hamiltonian reads [32]

$$
H_{\mathrm{ph}}=\sum_{\nu, k_{z}>0} \int d^{2} q \hbar \omega_{\mathbf{q}, k_{z}}\left[a_{\mathbf{q}, k_{z}}^{\nu}\right]^{\dagger} a_{\mathbf{q}, k_{z}}^{\nu},
$$

where $\omega_{\mathbf{q}, k_{z}}$ denotes the photon frequency. As mentioned earlier, we focus on cavity sizes such that only the photon with $k_{z}=\pi / L_{z}$ interacts strongly with the exciton, neglecting all the rest. For simplicity, we use $a_{\mathbf{q}}^{\nu}$ henceforth.

Considering in detail the microscopic coupling between excitons and photons [32], the polaritonic Hamiltonian in the basis of circularly polarized photons $\left\{b_{\mathbf{q}}^{-}, a_{\mathbf{q}}^{+}, b_{\mathbf{q}}^{+}, a_{\mathbf{q}}^{-}\right\}$ takes the form

$$
\mathcal{H}=\left[\begin{array}{cccc}
E_{\mathrm{ex}} & i \gamma & 0 & i \Gamma e^{2 i \varphi} \\
-i \gamma & E_{\mathrm{ph}} & i \Gamma e^{2 i \varphi} & 0 \\
0 & -i \Gamma e^{-2 i \varphi} & E_{\mathrm{ex}} & -i \gamma \\
-i \Gamma e^{-2 i \varphi} & 0 & i \gamma & E_{\mathrm{ph}}
\end{array}\right],
$$

where $E_{\mathrm{ex}}$ and $E_{\mathrm{ph}}$ are the bare exciton and photon energies, respectively, and the couplings read

$$
\begin{aligned}
& \gamma=\gamma_{0} \cos ^{2}(\theta / 2), \\
& \Gamma=\Gamma_{\text {ex }}+\gamma_{0} \sin ^{2}(\theta / 2) .
\end{aligned}
$$

Here, $\cos \theta=k_{z} / \sqrt{k_{z}^{2}+q^{2}}$ encodes the cavity size, and

$$
\begin{gathered}
\gamma_{0}=\frac{e \kappa \Delta}{\sqrt{\pi \hbar \omega_{\mathbf{k}} \epsilon_{0} L_{z}}}\left[F_{0}(\kappa)+F_{1}(\kappa) \frac{\hbar^{2} q^{2}}{\left(\Delta / v_{F}\right)^{2}}\right], \\
\Gamma_{\mathrm{ex}}=\frac{e \kappa \Delta}{\sqrt{\pi \hbar \omega_{\mathbf{k}} \epsilon_{0} L_{z}}} F_{2}(\kappa) \frac{\hbar^{2} q^{2}}{\left(\Delta / v_{F}\right)^{2}}
\end{gathered}
$$

to the order of $\mathcal{O}\left[\left(\hbar v_{F} q / \Delta\right)^{2}\right]$. Moreover, $e$ is the electron charge, $\epsilon_{0}$ is the vacuum permittivity, and $\kappa=a_{\mathrm{ex}} \Delta /\left(\hbar v_{F}\right)$. The real functions $F_{0}(\kappa), F_{1}(\kappa)$, and $F_{2}(\kappa)$ are given in Supplemental Material [32]. The Hamiltonian Eq. (4) contains the leading interactions in the system, although it neglects direct exciton coupling $[42,43]$ and the dielectric character of the cavity, which would yield a TE-TM splitting [44]. Our approach is complementary to that of Ref. [22], where the winding phase enters in a direct photon-photon coupling as a TE-TM splitting and is, therefore, proper of the cavity itself. We acknowledge the existence of more sources leading to this effect, e.g., a longitudinal-transverse splitting of the excitons [45]. However, such a detailed analysis—cf. Refs. [46,47]-is out of the scope of this work.

The results in Eqs. (5) show that the strength of the couplings $\gamma$ and $\Gamma$ is tunable with the cavity width. Whereas $\gamma$ is a mere renormalization of $\gamma_{0}, \Gamma$ contains two different contributions: The first, $\Gamma_{\mathrm{ex}}$, stems from the internal structure of excitons, particularly from the fact that they have a finite in-plane momentum; the second contains a term purely induced by the finite width of the cavity. At this stage, a qualitative yet illustrative characterization of the system is at reach in terms of the parameters $\gamma$ and $\Gamma$. The coupling $\gamma$ strongly hybridizes $\tau=+(-)$ excitons and $\nu=-(+)$ photons to yield four polariton bands, which are degenerate in pairs. A nonzero $\Gamma$ lifts the degeneracy between both lower polariton (LP) and upper polariton (UP) at $q \neq 0$, and it breaks the valley-polarization selection rule. Notice that the winding phases $e^{ \pm i 2 \varphi}$ provide the required $\ell_{z}=2$ angular momentum that allows the coupling of a photon and an exciton with $\tau=\nu$.

It is convenient to compare the Hamiltonian Eq. (4) and the model in Ref. [19]. In both models, the exciton-photon 


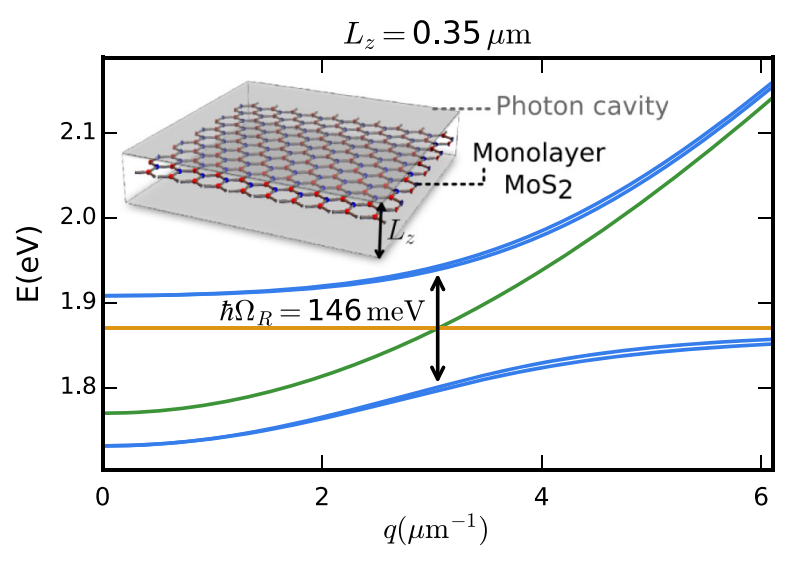

FIG. 1. Dispersion relation of a bare exciton and a photon in a cavity and the two resulting polaritonic branches in the strongcoupling regime.

couplings show a winding phase and have the same asymptotic limits for both small and large $q$ values. The calculation of the photon-exciton coupling, in our case, also takes into account the internal structure of the exciton. Furthermore, in Ref. [19], the use of a nonunitary transformation leads to a direct-photon coupling and an underestimation of the exciton-photon interaction in the strong-coupling regime $\left(q \sim k_{z}\right)$ [32].

In Fig. 1, we plot the dispersion relation for realistic values of the parameters of $\mathrm{MoS}_{2}$ excitons and photons in a microcavity of dimension $L_{z}=0.35 \mu \mathrm{m}$. In the strong-coupling regime, one finds that $\gamma \sim 10^{-2} \mathrm{eV}$ and $\Gamma_{\text {ex }} / \gamma_{0} \sim 10^{-8}$. For these parameters, $\Gamma_{\text {ex }}$ can be neglected. However, it might be significantly enhanced in systems with a higher $\hbar v_{F} q / \Delta$ ratio, or greater exciton radii, such as moiré patterns of graphene on top of an hBN substrate, where the gap ranges from 0 to $10 \mathrm{meV}$ [48]. On the other hand, we find that, within the strong-coupling regime, $k_{z} / q \sim 1$. Thus, according to Eq. (5), the finite size of the cavity results in a sizable $\Gamma$ and, therefore, plays a significant role in our system, as it is the principal source of selection-rule breaking. The fine splitting of the polariton bands is $\sim 5 \mathrm{meV}$ at strong coupling (see Fig. 1) and is probably too small to be measured at room temperature [18]. Yet, recent experiments on single-layer $\mathrm{MoSe}_{2}$ embedded in an optical cavity reported polariton Rabi splittings $\sim 20 \mathrm{meV}$, characterized by a $5 \mathrm{meV}$ linewidth at $4.2 \mathrm{~K}$, dominated mainly by photon losses [17]. The predicted splitting might therefore be observable at low temperatures in systems with greater $\Gamma$ values and by employing cavities realized with dielectric Bragg mirrors [49].

Berry curvature.-In order to analyze the dynamics of the polariton, we need to include also the intrinsic Berry phases of the constituents, the exciton and the photon. The $n$th eigenstate of a generic single-excitation Hamiltonian such as Eq. (4) takes the form $\left|\psi^{n}\right\rangle=\sum_{j} \psi_{j}^{n}\left[\hat{\phi}^{j}\right]^{\dagger}|0\rangle$, with $\mathcal{H} \psi_{n}=E_{n} \psi_{n}, \hat{\phi}^{j}$ second quantized operators describing the constituents and $|0\rangle$ the vacuum. When the state of the quasiparticle is not degenerate, we can study separately the Berry connection of each state $\left|\psi^{n}\right\rangle, \mathbf{A}^{n}=i\left\langle\psi^{n}\left|\nabla_{\mathbf{q}}\right| \psi^{n}\right\rangle$. We define the Berry connection of the bare constituents as $\mathbf{A}_{0}^{i j}=i\left\langle 0\left|\hat{\phi}^{i} \nabla_{\mathbf{q}}\left[\hat{\phi}^{j}\right]^{\dagger}\right| 0\right\rangle$. These definitions lead to

$$
\mathbf{A}^{n}=i\left[\psi^{n}\right]^{\dagger} \nabla_{\mathbf{q}} \psi^{n}+\left[\psi^{n}\right]^{\dagger} \mathbf{A}_{0} \psi^{n} .
$$

The BC can then be obtained as $\boldsymbol{\Omega}^{n}=\nabla_{\mathbf{q}} \times \mathbf{A}^{n}$. The form of Eq. (8) is independent of the choice of the basis states defined by $\hat{\phi}^{j}$. This analysis agrees with the results of Ref. [6], where the BC of excitons, regarded as composite particles, is shown to be composed by an intrinsic term due to the $\mathrm{BC}$ of conduction and valence band electrons, arising from the second term in Eq. (8), and an extrinsic one due to their coupling, coming from the first.

We first consider the intrinsic Berry curvature (IBC) of the exciton and the photon. We neglect the dependence of $\phi(q)$ on the center-of-mass momentum and assume equalmass conduction- and valence-band carriers. Then, the exciton $\mathrm{BC}$ is [6]

$$
\Omega_{\mathrm{ex}}^{\tau}(\mathbf{q})=\frac{1}{4} \sum_{\mathbf{q}^{\prime}}\left|\phi\left(q^{\prime}\right)\right|^{2} \sum_{\beta= \pm 1} \Omega_{c}^{\tau}\left(\mathbf{q}^{\prime}+\beta \mathbf{q} / 2\right),
$$

where $\Omega_{c}^{\tau}$ is the BC of the bare conduction electrons [32]. The Berry connection for circularly polarized photons reads $\mathbf{A}_{\text {ph }}^{\nu}(\mathbf{k})=\nu(\cos \theta-1) \mathbf{e}_{\phi}$, and the resulting $\mathrm{BC}$ acquires the simple form $\Omega_{\mathrm{ph}}^{\nu}(\mathbf{k})=\nu k_{z} / k^{3}$. As for the physical meaning of this $\mathrm{BC}$, it is not essentially different from that of other particles. As a matter of fact, Hall drifts have been predicted and observed at an interface between two materials; see Refs. $[9,10,50]$.

In the limit $\Gamma=0$, the two polaritonic branches are doubly degenerate, and we can label them with the valley index $\tau$. The UP and LP eigenstates are $\left|\psi_{\mathrm{UP}}^{\tau}\right\rangle=$ $\left(u_{q} a_{-\tau, \mathbf{q}}^{\dagger}+i \tau v_{q} b_{\tau, \mathbf{q}}^{\dagger}\right)|0\rangle$ and $\left|\psi_{\mathrm{LP}}^{\tau}\right\rangle=\left(i \tau v_{q} a_{-\tau, \mathbf{q}}^{\dagger}+u_{q} b_{\tau, \mathbf{q}}^{\dagger}\right)|0\rangle$, respectively, with $u_{q}$ and $v_{q}$ real normalized amplitudes. Remarkably, polaritons show a finite $\mathrm{BC}$ that is due to the intrinsic contributions of the constituents, being proportional to the probabilities $u_{q}^{2}$ and $v_{q}^{2}$. For UPs, we have

$\Omega_{\mathrm{UP}}^{\tau}=u_{q}^{2} \Omega_{\mathrm{ph}}^{-\tau}+v_{q}^{2} \Omega_{\mathrm{ex}}^{\tau}+\left(\mathbf{A}_{\mathrm{ph}}^{-\tau}-\mathbf{A}_{\mathrm{ex}}^{\tau}\right) \cdot \hat{z} \times \nabla_{\mathbf{q}} u_{q}^{2}$,

and analogously for LPs with $u_{q}$ and $v_{q}$ interchanged. Clearly, the BC of the composite system is significantly enhanced by the coupling, as the extrinsic term containing $\nabla_{\mathbf{q}} u_{q}^{2}$ peaks in the strong-coupling regime [51].

For $\Gamma \neq 0$, the polariton branches split. The new eigenfunctions hybridize excitons from the two valleys and photons with opposite polarizations with equal amplitude, and the Berry curvature of each quasiparticle vanishes. For the extrinsic Berry curvature (EBC), an explicit calculation shows that it also vanishes for all $\mathbf{q} \neq 0$. However, the UPs and LPs are degenerate at $\mathbf{q}=0$, giving rise to a $\delta^{(2)}(\mathbf{q})$ 
structure of the BC. A nonzero Berry curvature arises if time-reversal symmetry is broken, and a magnetic field induces a Zeeman coupling [19,27]. Alternatively, a polariton Hall current will exist when the initial state is given by a finite population of chiral excitations, which can be created using circularly polarized light.

In the presence of a Zeeman coupling $V_{z}$, the $\mathrm{BC}$ is smeared around small momenta. A simple effective Hamiltonian for either the UP $(s=1)$ or the LP $(s=-1)$ branches can be obtained by treating $\gamma$ and $\Gamma$ as perturbative parameters and considering $V_{z} \ll\left|E_{\mathrm{ph}}-E_{\mathrm{ex}}\right|$. At small momenta,

$$
H_{\mathrm{eff}}^{s}=f_{s}(q)+\left(\begin{array}{cc}
\Delta_{s}(q) & \alpha q^{2} e^{2 i \phi} \\
\alpha^{*} q^{2} e^{-2 i \phi} & -\Delta_{s}(q)
\end{array}\right),
$$

with $\alpha=-4 \gamma \tilde{\Gamma} /\left(E_{\mathrm{ex}}-E_{\mathrm{ph}}\right), \tilde{\Gamma}=\Gamma / q^{2}$, and $f_{s}$ and $\Delta_{s} \propto V_{z}$ given in Supplemental Material [32]. The correspondence between the polaritonic branches and gapped (gapless for $V_{z}=0$ ) bilayer graphene [52] becomes manifest here, providing us with a qualitative understanding of the EBC. The $\mathrm{BC}$ then reads

$$
\Omega_{\mathrm{pol}}^{s, \lambda}(\mathbf{q})=\frac{2 \lambda \Delta_{s}}{\alpha} \frac{q^{2}}{\left[q^{2}+\left(\Delta_{s} / \alpha\right)^{2}\right]^{3 / 2}} .
$$

The $\mathrm{BC}$ for a generic parameter regime and $V_{z}=\gamma / 5$ is shown in Fig. 2 for one UP and one LP branch, the BC in the two other bands having the opposite sign. A breakdown of the different terms in Eq. (8) is presented therein. We can distinguish two different regions within the strong-coupling regime where either the intrinsic or the extrinsic contributions to $\Omega$ dominate. For $q \rightarrow 0$, the EBC goes to zero, whereas the IBC remains finite (see the inset in Fig. 2). In that range of momenta, the polariton behaves as merely inheriting the $\mathrm{BC}$ of its constituents.

Remarkably, it turns out that the $\mathrm{BC}$ of excitons is 6 orders of magnitude smaller than that of photons and, therefore, can be neglected. This fact allows the interpretation of the dotted curves in Fig. 2 in simple terms: A greater photonic component in the polaritonic eigenstate yields a higher value of the IBC. This behavior combines with the decay of $\Omega_{\mathrm{ph}}$ with $q$ to make the IBC peak at $q=0$ for LPs and at $q>0$ for UPs.

As for the dominance of the EBC, it happens near the crossing between the bare exciton and the photon bands. It becomes an order of magnitude greater than its counterpart for UPs and around twice as much as the IBC for the LPs. There is a significant difference in the $\mathrm{BC}$ between the UP and LP branches: The former reaches higher absolute values and peaks at larger momenta. Once more, these features can be understood in terms of the competition between two factors. First, the proximity in energy between a pair of either UP or LP branches is expected to increase the value of the EBC. Notice that UP dispersion relations come closer to each other with $q$, whereas the opposite (a)

Polariton Hall effect

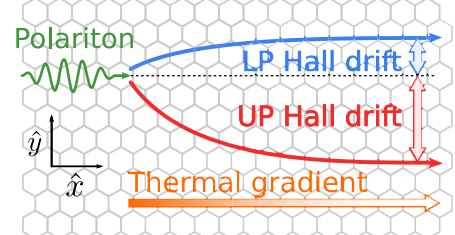

(b) Spectrum

(c)

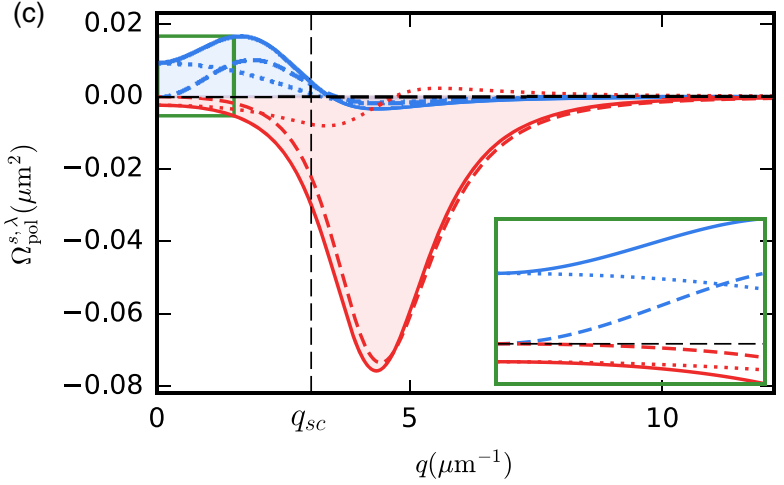

FIG. 2. (a) Schematics of the Hall drift experienced by LP and UP upon application of a thermal gradient. (b) Zeeman split polariton spectrum. (c) Extrinsic (dashed line), intrinsic (dotted line), and total (solid line) BC for the UP of highest (red) and the LP of lowest (blue) energy. Dashed black lines are a guide to the eye, with $q_{s c}$ such that $E_{\mathrm{ex}}\left(q_{s c}\right)=E_{\mathrm{ph}}\left(q_{s c}\right)$. The lower inset enlarges the region of small momenta that appears squared in green. The model of Eq. (4) and the Berry connection given by Eq. (8) were used in this section.

happens with LPs' see Fig. 2(b). Second, the EBC is expected to peak at momenta where the coupling is the greatest. As a result of this balance, the location of the EBC peaks in Fig. 2, which shift away from $q_{s c}$, can be explained. We emphasized that photons are the only significant source of $\mathrm{BC}$ for polaritons either via the IBC or by means of the strong coupling with matter.

Polariton anomalous Hall effect.-The BC manifests in a Hall current perpendicular to an applied in-plane force. On a general basis, assuming a polaritonic wave packet in the $s$ branch and the $\lambda$ split band, centered at $\mathbf{r}_{c}=\left(x_{c}, y_{c}\right)$ and $\mathbf{q}_{c}=\left(q_{c, x}, q_{c, y}\right)$ and with energy $E_{\mathrm{pol}}^{s, \lambda}\left(\mathbf{r}_{c}, \mathbf{q}_{c}\right)$, we can describe the evolution of its coordinates by semiclassical equations of motion that include the $\mathrm{BC}$ through an anomalous velocity [53,54]:

$$
\dot{\mathbf{r}}_{c}=\frac{\partial E_{\mathrm{pol}}^{s, \lambda}}{\partial \mathbf{q}_{c}}-\dot{\mathbf{q}}_{c} \times \boldsymbol{\Omega}_{\mathrm{pol}}^{s, \lambda}\left(q_{c}\right), \quad \dot{\mathbf{q}}_{c}=-\frac{\partial E_{\mathrm{pol}}^{s, \lambda}}{\partial \mathbf{r}_{c}} .
$$

Because of the anomalous term, a polariton anomalous Hall effect naturally arises when hybrid modes are accelerated. An anomalous exciton current was observed in Ref. [12], where the nonzero $\dot{\mathbf{q}}_{c}$ was provided by a thermal gradient applied to the sample. The same scheme can be applied to polaritons in the strong-coupling regime. Further alternatives to achieve wave packet accelerations, like a gradient in the cavity thickness [55], can be also borne in mind regarding experimental realizations. 
We now consider a polaritonic wave packet initially centered at $\mathbf{r}_{c}=\mathbf{q}_{c}=0$ under the influence of an effective force in the $\hat{x}$ direction; Eqs. (13) predict for the Hall drift

$$
y_{c}=\int_{0}^{q_{c}} d q_{c}^{\prime} \Omega_{\mathrm{pol}}^{s, \lambda}\left(q_{c}^{\prime}\right),
$$

where the angular symmetry of $E_{\text {pol }}^{s, \lambda}\left(\mathbf{r}_{c}, \mathbf{q}_{c}\right)$ has been taken into account. Remarkably, the result applies also in the case of relativistic corrections that arise due to the coupling with photons. Assuming the domain of integration to exceed the strong-coupling regime, we extend the integration to infinity, so that the area under the curves in Fig. 2 gives a good approximation of the polariton drift. For realistic values of the parameters, we obtain $y_{c} \simeq 0.2 \mu \mathrm{m}$ for UPs and $y_{c} \simeq 0.03 \mu \mathrm{m}$ for LPs. Note that the IBC drives a drift that amounts only to $\sim 10^{-2} \mu \mathrm{m}$.

Regarding a possible realization of our predictions, we emphasize that the generation and observation of polaritonic wave packets are already at experimental reach. Techniques like optical injection with laser beams [56] and polarized short pulses [57], direction-controlled ballistic ejection from condensates [58], or out-of-resonance pumping in TMDs [59] provide very localized wave packets, to the extent of approaching the Heisenberg limit [60], micron-scale propagation lengths, and trajectories that can be tracked in real time $[57,61,62]$.

Conclusion.-We have presented a microscopic treatment of the exciton-photon coupling in a cavity-embedded $\mathrm{MoS}_{2}$ monolayer, focusing on the spectrum and Berry curvature. Remarkably, we find that the cavity size promotes a selection-rule breaking by a valley-polarization crosscoupling, characterized by a winding phase. The coupling results in a splitting of the polaritonic branches and a strong enhancement of the polariton $\mathrm{BC}$, much beyond their constituent contributions. The polariton $\mathrm{BC}$ peaks in the strong-coupling regime and gives rise to a polariton anomalous Hall effect, thus promoting 2D materials as a platform to study the topology of hybrid light-matter states.

A. G.-R., L. M.-M., and F. G. acknowledge the European Commission under the Graphene Flagship, Contract No. CNECTICT-604391. L. C. and F. G. acknowledge funding from the European Union's Seventh Framework Program (FP7/2007-2013) through the ERC Advanced Grant NOVGRAPHENE (Grant Award No. 290846), and L. C. acknowledges the Comunidad de Madrid through Grant No. MAD2D-CM, S2013/MIT-3007. L. M.-M. and F. J. G.-V. acknowledge financial support by the Spanish MINECO under Contract No. MAT2014-53432-C5.

\footnotetext{
*Corresponding author. angelgutierrezrubio@gmail.com

${ }^{\dagger}$ Corresponding author. luca.chirolli@gmail.com
}

[1] D. Xiao, M.-C. Chang, and Q. Niu, Rev. Mod. Phys. 82, 1959 (2010).

[2] M. V. Berry, Proc. R. Soc. A 392, 45 (1984).

[3] D. J. Thouless, M. Kohmoto, M. P. Nightingale, and M. den Nijs, Phys. Rev. Lett. 49, 405 (1982).

[4] Q. Niu, D. J. Thouless, and Y.-S. Wu, Phys. Rev. B 31, 3372 (1985).

[5] F. D. M. Haldane and S. Raghu, Phys. Rev. Lett. 100, 013904 (2008).

[6] W. Yao and Q. Niu, Phys. Rev. Lett. 101, 106401 (2008).

[7] J. ichi Igarashi, J. Phys. Soc. Jpn. 57, 962 (1988).

[8] J. C. W. Song and M. S. Rudner, Proc. Natl. Acad. Sci. U.S.A. 113, 4658 (2016).

[9] M. Onoda, S. Murakami, and N. Nagaosa, Phys. Rev. Lett. 93, 083901 (2004).

[10] O. Hosten and P. Kwiat, Science 319, 787 (2008).

[11] E. Estrecho, T. Gao, S. Brodbeck, M. Kamp, C. Schneider, S. Höfling, A. G. Truscott, and E. A. Ostrovskaya, Sci. Rep. 6, 37653 (2016).

[12] M. Onga, Y. Zhang, T. Ideue, and Y. Iwasa, Nat. Mater. 16, 1193 (2017).

[13] J. A. Hutchison, T. Schwartz, C. Genet, E. Devaux, and T. W. Ebbesen, Angew. Chem. 124, 1624 (2012).

[14] R. Roldan, L. Chirolli, E. Prada, J. A. Silva-Guillen, P. San-Jose, and F. Guinea, Chem. Soc. Rev. 46, 4387 (2017).

[15] N. Lundt, S. Klembt, E. Cherotchenko, S. Betzold, O. Iff, A. V. Nalitov, M. Klaas, C. P. Dietrich, A. V. Kavokin, S. Höfling, and C. Schneider, Nat. Commun. 7, 13328 (2016).

[16] X. Liu, T. Galfsky, Z. Sun, F. Xia, E.-c. Lin, Y.-H. Lee, S. Kéna-Cohen, and V. M. Menon, Nat. Photonics 9, 30 (2015).

[17] S. Dufferwiel, S. Schwarz, F. Withers, A. A. P. Trichet, F. Li, M. Sich, O. Del Pozo-Zamudio, C. Clark, A. Nalitov, D. D. Solnyshkov, G. Malpuech, K. S. Novoselov, J. M. Smith, M. S. Skolnick, D. N. Krizhanovskii, and A. I. Tartakovskii, Nat. Commun. 6, 8579 (2015).

[18] S. Wang, S. Li, T. Chervy, A. Shalabney, S. Azzini, E. Orgiu, J. A. Hutchison, C. Genet, P. Samorì, and T. W. Ebbesen, Nano Lett. 16, 4368 (2016).

[19] T. Karzig, C.-E. Bardyn, N. H. Lindner, and G. Refael, Phys. Rev. X 5, 031001 (2015).

[20] A. V. Nalitov, G. Malpuech, H. Terças, and D. D. Solnyshkov, Phys. Rev. Lett. 114, 026803 (2015).

[21] C.-E. Bardyn, T. Karzig, G. Refael, and T. C. H. Liew, Phys. Rev. B 91, 161413 (2015).

[22] O. Bleu, G. Malpuech, Y. Gao, and D. D. Solnyshkov, Phys. Rev. Lett. 121, 020401 (2018).

[23] H. Zeng, J. Dai, W. Yao, D. Xiao, and X. Cui, Nat. Nanotechnol. 7, 490 (2012).

[24] K. F. Mak, K. He, J. Shan, and T. F. Heinz, Nat. Nanotechnol. 7, 494 (2012).

[25] E. Prada, J. V. Alvarez, K. L. Narasimha-Acharya, F. J. Bailen, and J. J. Palacios, Phys. Rev. B 91, 245421 (2015).

[26] T. C. Berkelbach, M. S. Hybertsen, and D. R. Reichman, Phys. Rev. B 88, 045318 (2013).

[27] A. V. Stier, K. M. McCreary, B. T. Jonker, J. Kono, and S. A. Crooker, Nat. Commun. 7, 10643 (2016).

[28] A. Srivastava and A. Imamoğlu, Phys. Rev. Lett. 115, 166802 (2015).

[29] J. Zhou, W.-Y. Shan, W. Yao, and D. Xiao, Phys. Rev. Lett. 115, 166803 (2015) 
[30] H. Rostami and R. Asgari, Phys. Rev. B 91, 075433 (2015).

[31] H.-P. Komsa and A. V. Krasheninnikov, Phys. Rev. B 86, 241201 (2012).

[32] See Supplemental Material at http://link.aps.org/ supplemental/10.1103/PhysRevLett.121.137402 for further details concerning the microscopic derivation of the Hamiltonian and the discussion about topology, which includes Refs. [33-41].

[33] D. Xiao, G.-B. Liu, W. Feng, X. Xu, and W. Yao, Phys. Rev. Lett. 108, 196802 (2012).

[34] K. F. Mak, K. L. McGill, J. Park, and P. L. McEuen, Science 344, 1489 (2014).

[35] I. Garate and M. Franz, Phys. Rev. B 84, 045403 (2011).

[36] D. K. Efimkin and Y. E. Lozovik, Phys. Rev. B 87, 245416 (2013).

[37] D. Xiao, W. Yao, and Q. Niu, Phys. Rev. Lett. 99, 236809 (2007).

[38] P. San-Jose, V. Parente, F. Guinea, R. Roldán, and E. Prada, Phys. Rev. X 6, 031046 (2016).

[39] W. Liu, B. Lee, C. H. Naylor, H.-S. Ee, J. Park, A. T. C. Johnson, and R. Agarwal, Nano Lett. 16, 1262 (2016).

[40] S. Bravyi, D. P. DiVincenzo, and D. Loss, Ann. Phys. (Amsterdam) 326, 2793 (2011).

[41] P. Coleman, Introduction to Many-Body Physics (Cambridge University Press, Cambridge, England, 2015).

[42] T. Yu and M. W. Wu, Phys. Rev. B 89, 205303 (2014).

[43] H. Yu, G.-B. Liu, P. Gong, X. Xu, and W. Yao, Nat. Commun. 5, 3876 (2014).

[44] G. Panzarini, L. C. Andreani, A. Armitage, D. Baxter, M. S. Skolnick, V. N. Astratov, J. S. Roberts, A. V. Kavokin, M. R. Vladimirova, and M. A. Kaliteevski, Phys. Rev. B 59, 5082 (1999).

[45] M.Z. Maialle, E. A. de Andrada e Silva, and L. J. Sham, Phys. Rev. B 47, 15776 (1993).

[46] I. A. Shelykh, G. Pavlovic, D. D. Solnyshkov, and G. Malpuech, Phys. Rev. Lett. 102, 046407 (2009).

[47] O. Bleu, D. D. Solnyshkov, and G. Malpuech, Phys. Rev. B 96, 165432 (2017).

[48] P. San-Jose, A. Gutiérrez-Rubio, M. Sturla, and F. Guinea, Phys. Rev. B 90, 115152 (2014).
[49] A. V. Kavokin, I. A. Shelykh, and G. Malpuech, Phys. Rev. B 72, 233102 (2005).

[50] C. Prajapati, D. Ranganathan, and J. Joseph, Opt. Lett. 38, 2459 (2013).

[51] It may seem that the BC as described by Eq. (10) is not gauge invariant, in that the Berry connection can be defined up to the gradient of a scalar function. However, as pointed out in the section Berry curvature, a redefinition of the wave function of the constituents affects both their Berry connection and their coupling, in a way that the result is basis independent.

[52] A. H. Castro Neto, F. Guinea, N. M. R. Peres, K. S. Novoselov, and A. K. Geim, Rev. Mod. Phys. 81, 109 (2009).

[53] M. C. Chang and Q. Niu, Phys. Rev. Lett. 75, 1348 (1995).

[54] N. A. Sinitsyn, J. Phys. Condens. Matter 20, 023201 (2008).

[55] B. Sermage, G. Malpuech, A. V. Kavokin, and V. ThierryMieg, Phys. Rev. B 64, 081303 (2001).

[56] D. Ballarini, M. De Giorgi, E. Cancellieri, R. Houdre, E. Giacobino, R. Cingolani, A. Bramati, G. Gigli, and D. Sanvitto, in Proceedings of the 2013 Conference on Lasers and Electro-Optics Europe and International Quantum Electronics Conference CLEO EUROPE/IQEC (IEEE, New York, 2013).

[57] C. Adrados, T. C. H. Liew, A. Amo, M. D. Martín, D. Sanvitto, C. Antón, E. Giacobino, A. Kavokin, A. Bramati, and L. Viña, Phys. Rev. Lett. 107, 146402 (2011).

[58] T. Gao, P. S. Eldridge, T. C. H. Liew, S. I. Tsintzos, G. Stavrinidis, G. Deligeorgis, Z. Hatzopoulos, and P. G. Savvidis, Phys. Rev. B 85, 235102 (2012).

[59] D. Sanvitto and S. Kéna-Cohen, Nat. Mater. 15, 1061 (2016).

[60] H. Deng, H. Haug, and Y. Yamamoto, Rev. Mod. Phys. 82, 1489 (2010).

[61] G. G. Rozenman, K. Akulov, A. Golombek, and T. Schwartz, ACS Photonics 5, 105 (2018).

[62] G. Lerario, D. Ballarini, A. Fieramosca, A. Cannavale, A. Genco, F. Mangione, S. Gambino, L. Dominici, M. De Giorgi, G. Gigli, and D. Sanvitto, Light Sci. Appl. 6, e16212 (2017). 